“(C) 2015 IEEE. Personal use of this material is permitted. Permission from IEEE must be obtained for all other uses, in any current or future media, including reprinting/republishing this material for advertising or promotional purposes, creating new collective works, for resale or redistribution to servers or lists, or reuse of any copyrighted component of this work in other works." 


\title{
Anti-Noise-Folding Regularized Subspace Pursuit Recovery Algorithm for Noisy Sparse Signals
}

\author{
Xianjun Yang $^{\dagger *}$, Qimei Cui*, Eryk Dutkiewicz ${ }^{\dagger}$, Xiaojing Huang $^{\ddagger}$, Xiaofeng Tao*, Gengfa Fang ${ }^{\dagger}$ \\ *Wireless Technology Innovation Institute, Key Lab. of Universal Wireless Communication, Ministry of Education, \\ Beijing University of Posts and Telecommunications, Beijing, China. \\ ${ }^{\dagger}$ Department of Electronic Engineering, Macquarie University, Sydney, Australia. \\ ${ }^{\ddagger}$ CSIRO ICT Center, Sydney, Australia. \\ E-mail: xianjun.yang@mq.edu.au
}

\begin{abstract}
Denoising recovery algorithms are very important for the development of compressed sensing (CS) theory and its applications. Considering the noise present in both the original sparse signal $x$ and the compressive measurements $y$, we propose a novel denoising recovery algorithm, named Regularized Subspace Pursuit (RSP). Firstly, by introducing a data pre-processing operation, the proposed algorithm alleviates the noise-folding effect caused by the noise added to $x$. Then, the indices of the nonzero elements in $x$ are identified by regularizing the chosen columns of the measurement matrix. Afterwards, the chosen indices are updated by retaining only the largest entries in the Minimum Mean Square Error (MMSE) estimated signal. Simulation results show that, compared with the traditional orthogonal matching pursuit (OMP) algorithm, the proposed RSP algorithm increases the successful recovery rate (and reduces the reconstruction error) by up to $50 \%$ and $86 \%(35 \%$ and $65 \%$ ) in high noise level scenarios and inadequate measurements scenarios, respectively.
\end{abstract}

\section{INTRODUCTION}

In recent years, Compressed Sensing (CS) [1] [2] has been a hot research topic in the signal processing field and has been widely applied in many areas such as imaging, sub-Nyquist sampling, wireless sensor networks and so on [3]. In most of the realistic applications, the compressive measurements are contaminated by noise, i.e., the signal model is

$$
\mathbf{y}=\mathbf{A x}+\mathbf{w}
$$

where $\mathbf{x}$ is an $N \times 1$ unknown $K$-sparse signal, i.e., only $K$ elements of $\mathbf{x}$ are nonzero, where the indices of the $K$ elements are termed the signal support $\Lambda$ of $\mathbf{x}, \mathbf{A}$ is an $M \times N$ Gaussian or Bernoulli distributed measurement matrix, which satisfies the Restricted Isometry Property (RIP) [4], y is an $M \times 1$ measurement vector, and $\mathbf{w}$ is an $M \times 1$ Gaussian white noise vector, where each element has mean 0 and variance $\sigma_{w}^{2}$.

However, in many practical scenarios, e.g., the application of CS into the design of sub-Nyquist sampling devices [5] [6], the sparse signal is contaminated by noise prior to measurement. Thus, in this paper, we will consider the following more general signal model

$$
\mathbf{y}=\mathbf{A}(\mathbf{x}+\mathbf{z})+\mathbf{w}=\mathbf{A} \mathbf{x}+\mathbf{v}
$$

where $\mathbf{v}=\mathbf{A} \mathbf{z}+\mathbf{w}$, and $\mathbf{z}$ is an $N \times 1$ Gaussian white noise vector, where each element has mean 0 and variance $\sigma_{z}^{2}$, and we assume that $\sigma_{w}^{2}=\sigma_{z}^{2}=\sigma^{2}$ for simplicity. It is worth mentioning that the pre-measurement noise $\mathbf{z}$ results in much larger noise power in the compressive measurements $\mathbf{y}$ than that in the original noisy sparse signal, which is called the noise-folding effect [7]. The reason for the noise-folding effect is that the measurement matrix $\mathbf{A}$ combines all the noise elements in $\mathbf{z}$, even those corresponding to the zero elements in $\mathbf{x}$.

Reliable and stable CS recovery algorithms are one of the key components of CS theory and are very important for the application of CS in noisy scenarios. Thus, in this paper, we aim to develop an efficient CS denoising algorithm to reconstruct the noise-contaminated sparse signals from noisy compressive measurements with high successful recovery rate.

\section{A. Related Work}

The existing denoising CS recovery algorithms can be generally classified into the following two categories.

1) Regularization Methods: A natural approach to finding the sparsest signal satisfying (2) is to solve the following $\ell_{0}$ regularization problem

$$
\hat{\mathbf{x}}=\arg \min _{\mathbf{x} \in \mathbb{R}^{N}}\left\{\|\mathbf{y}-\mathbf{A x}\|_{2}^{2}+\lambda\|\mathbf{x}\|_{0}\right\},
$$

where $\lambda>0$ is a regularization parameter, the $\ell_{0}$ quasi-norm $\|\mathbf{x}\|_{0}$ counts the number of the nonzero components in $\mathbf{x}$, and the $\ell_{p}$ norm for $p>0$ is defined as $\|\mathbf{x}\|_{p}=\left(\sum_{i=1}^{N}\left|x_{i}\right|^{p}\right)^{1 / p}$.

However, the $\ell_{0}$ regularization problem is NP-hard. To overcome this obstacle, problem (3) is relaxed to the following $\ell_{p}$ regularization problem

$$
\hat{\mathbf{x}}=\arg \min _{\mathbf{x} \in \mathbb{R}^{N}}\left\{\|\mathbf{y}-\mathbf{A x}\|_{2}^{2}+\lambda\|\mathbf{x}\|_{p}^{p}\right\},
$$

where $0<p \leq 1$. When $p=1$, problem (4) is convex and is called $\ell_{1}$ regularization [8], which has good recovery performance. When $0<p<1$, problem (4) is non-convex and difficult to solve, but it has better recovery performance than $\ell_{1}$ regularization. Recently, efficient algorithms to resolve the above non-convex problems have been proposed. For example, authors in [9] have proposed a fast iterative half thresholding algorithm for the $\ell_{1 / 2}$ regularization problem. 
2) Greedy Algorithms: Orthogonal Matching Pursuit (OMP) [10] is a representative greedy CS recovery algorithm for noise-free scenarios. The OMP algorithm begins by initializing the estimated signal support set $\Lambda_{0}$ as an empty set $\emptyset$ and the residual $\mathbf{r}_{0}$ as $\mathbf{y}$. At the $t^{t h}$ iteration, the OMP algorithm finds one single column of matrix $\mathbf{A}$ that is most highly correlated with the residual in the $(t-1)^{t h}$ iteration $\mathbf{r}_{t-1}$, i.e., the index of this column $\lambda_{t}$ is obtained by

$$
\lambda_{t}=\arg \max _{j=1, \ldots, N}\left|\left\langle\mathbf{r}_{t-1}, \mathbf{a}_{j}\right\rangle\right|,
$$

where $\left\langle\mathbf{r}_{t-1}, \mathbf{a}_{j}\right\rangle$ is the inner product of $\mathbf{r}_{t-1}$ and $\mathbf{a}_{j}$, with $\mathbf{a}_{j}$ being the $j^{t h}$ column of matrix $\mathbf{A}$. Then, the index $\lambda_{t}$ is added to the support set by $\Lambda_{t}=\Lambda_{t-1} \cup\left\{\lambda_{t}\right\}$. With the leastsquare (LS) estimation method, the estimated signal at the $t^{t h}$ iteration $\hat{\mathbf{x}}_{t}$ is calculated by

$$
\hat{\mathbf{x}}_{t}=\left\{\begin{array}{ll}
\mathbf{A}_{\Lambda_{t}}^{\dagger} \mathbf{y}, & \text { on the support set } \Lambda_{t} \\
\mathbf{0}, & \text { elsewhere }
\end{array},\right.
$$

where $\mathbf{A}_{\Lambda_{t}}$ consists of the columns of matrix $\mathbf{A}$ which are indexed by $\Lambda_{t}, \mathbf{A}_{\Lambda_{t}}^{\dagger}$ is the Moore-Penrose pseudo-inverse of $\mathbf{A}_{\Lambda_{t}}$. Then, the residual at the $t^{t h}$ iteration is expressed as

$$
\mathbf{r}_{t}=\mathbf{y}-\mathbf{A} \hat{\mathbf{x}}_{t} .
$$

After $K$ iterations, the reconstructed signal is $\hat{\mathbf{x}}_{K}$ and the reconstructed signal support is $\Lambda_{K}$.

Recently, several methods have been proposed to improve the recovery performance of OMP in noisy scenarios. Firstly, regularized OMP (ROMP) [11] was proposed to incorporate the property of convex relaxation by selecting only the comparable coordinates of the relevance vector $\mathbf{A}^{H} \mathbf{r}_{t-1}$. Then, CoSaMP [12] was proposed to reduce the effect of noise by pruning the LS estimated signal. Subsequently, Subspace Pursuit (SP) [13] was proposed to achieve a similar reconstruction accuracy as convex relaxation by obtaining a $K$-dimensional hyperplane closer to $\mathbf{y}$ after each iteration. Meanwhile, it has been proved that, under the conditions of the minimum magnitude of the nonzero elements in $\mathrm{x}$ and the mutual coherence or RIP, OMP can exactly reconstruct the support of the sparse signal from noisy measurements with high probability [14] [15].

\section{B. Our Contributions}

Since greedy algorithms are much faster and easier to implement than regularization methods, this paper focuses on the improvements of greedy algorithms in noisy environments. The existing denoising greedy algorithms improve OMP either from the angle of finding indices of nonzero elements, e.g., ROMP, or the angle of signal estimation, e.g., CoSaMP and SP. However, none of them improves OMP from both angles.

Thus, this paper proposes a Regularized Subspace Pursuit (RSP) algorithm to improve OMP from both aspects mentioned above simultaneously. Firstly, the proposed RSP algorithm alleviates the noise-folding effect by introducing a data pre-processing operation. Secondly, the signal support $\Lambda$ is identified by regularizing the chosen columns of $\mathbf{A}$, and then the chosen indices are updated by retaining only the largest entries of the estimated signal. Thirdly, instead of using LS method, Minimum Mean Square Error (MMSE) estimation is adopted to further reduce the effect of noise.

Simulation results show that, compared with the existing OMP, ROMP, CoSaMP and SP algorithms, the proposed RSP algorithm has the highest successful recovery rate and the smallest reconstruction error when the noise variance is high and the number of measurements is not large enough. For example, compared with the OMP algorithm, the proposed RSP algorithm increases the successful recovery rate (reduces the normalized reconstruction error) by up to $50 \%$ and $86 \%$ (35\% and 65\%) in high noise level scenarios and inadequate measurements scenarios, respectively.

The rest of this paper is organized as follows. Section II describes the proposed RSP algorithm. Section III evaluates the recovery performance of RSP in comparison with existing OMP, ROMP, CoSaMP and SP algorithms. Finally, Section IV concludes this paper.

\section{Proposed RSP Algorithm}

Based on the traditional OMP algorithm and motivated by ROMP and SP algorithms, we propose the RSP algorithm to combat the effects of both the pre-measurement noise $\mathbf{z}$ and the measurement noise $\mathbf{w}$.

\section{A. Basic Idea}

Five key parts of RSP algorithm are stated as follows.

1) Pre-processing Operation: The covariance matrix of the equivalent noise vector $\mathbf{v}$ in (2) is expressed as

$$
\mathbf{Q}=\sigma^{2}\left(\mathbf{I}+\mathbf{A} \mathbf{A}^{T}\right) .
$$

Thus, the equivalent noise $\mathbf{v}$ is not white any more unless the matrix $\mathbf{A A}^{T}$ is proportional to the identity matrix $\mathbf{I}$. One example of the above exception is that matrix $\mathbf{A}$ is a concatenation of $p=\frac{N}{M}$ orthonormal matrices, i.e., $\mathbf{A}=$ $\left[\mathbf{A}^{(1)}, \ldots, \mathbf{A}^{(p)}\right]$, where the dimension of $\mathbf{A}^{(k)}$ is $M \times M$. Then, we have $\mathbf{A} \mathbf{A}^{T}=p \mathbf{I}$, and $\mathbf{Q}=\left(1+\frac{N}{M}\right) \sigma^{2} \mathbf{I}$. This is a special case of the noise-folding effect, where the variance of the equivalent additive white noise is increased $\frac{N}{M}$ times.

In the proposed RSP algorithm, to better reconstruct the sparse signal, the noise $\mathbf{v}$ is whitened by multiplying $\mathbf{Q}^{-1 / 2}$ at both sides of (2), then we obtain

$$
\tilde{\mathbf{y}}=\mathbf{B x}+\mathbf{u}
$$

where $\tilde{\mathbf{y}}=\mathbf{Q}^{-1 / 2} \mathbf{y}, \mathbf{B}=\mathbf{Q}^{-1 / 2} \mathbf{A}$ and $\mathbf{u}=\mathbf{Q}^{-1 / 2} \mathbf{v}$. Now, the new equivalent noise $\mathbf{u}$ is white and has variance $\sigma^{2}$.

2) Scaled Relevance Vector: Let $\mathbf{b}_{j}$ denote the $j^{\text {th }}$ column of the new measurement matrix $\mathbf{B}$, and $\left\|\mathbf{b}_{j}\right\|_{2}$ represent the $\ell_{2}$ norm of $\mathbf{b}_{j}$. To make sure that the differences among $\left\|\mathbf{b}_{j}\right\|_{2}$ do not affect the precision in finding the index with the biggest relevance from the relevance vector $\mathbf{B}^{H} \mathbf{r}_{t-1}$, we propose to adopt the scaled relevance vector $\mathbf{u}_{t}$, where the $j^{t h}$ element of $\mathbf{u}_{t}$ is expressed as

$$
\mathbf{u}_{t}(j)=\boldsymbol{\beta}(j) / \boldsymbol{\theta}(j),
$$

where $\boldsymbol{\beta}=\mathbf{B}^{H} \mathbf{r}_{t-1}$ and $\boldsymbol{\theta}=\sqrt{\operatorname{diag}\left(\mathbf{B}^{H} \mathbf{B}\right)}$. 
3) Identifying the Indices by Regularization: According to the RIP property [4], every $K$ columns of matrix $\mathbf{B}$ constitute an approximate orthonormal matrix. At the $t^{\text {th }}$ iteration, to make the identified columns of $\mathbf{B}$ behave like an orthonormal matrix, the identified columns are regularized via choosing a subset $J_{0}$ of the $K$ largest indices in $\mathbf{u}_{t}$ by letting

$$
J_{0}=\left\{j \mid \mathbf{u}_{t}(j) \geq u_{t, \max } / 2\right\},
$$

where $u_{t, \max }=\max _{j}\left[\mathbf{u}_{t}(j)\right]$ is the maximum element of $\mathbf{u}_{t}$. Then, the set $J_{0}$ is augmented to the identified indices in the previous iteration by letting $\Lambda_{t}=\Lambda_{t-1} \cup\left\{J_{0}\right\}$.

4) Estimating the Signal with MMSE: Since the LS method used in the existing greedy algorithms amplifies the noise, we utilize the denoising MMSE method to estimate the signal $\mathbf{x}_{\Lambda_{t}}$ from the following problem

$$
\tilde{\mathbf{y}}=\mathbf{B}_{\Lambda_{t}} \mathbf{x}_{\Lambda_{t}}+\mathbf{u}
$$

where $\mathbf{x}_{\Lambda_{t}}$ contains the elements of $\mathbf{x}$ indexed by $\Lambda_{t}$. As we know, the MMSE method requires to know the power of $\mathbf{x}_{\Lambda_{t}}$, which varies with the change of $\Lambda_{t}$. Thus, we propose to estimate the power of $\mathbf{x}_{\Lambda_{t}}$ with the LS method, i.e.,

$$
P=\left(\mathbf{B}_{\Lambda_{t}}^{\dagger} \tilde{\mathbf{y}}\right)^{H}\left(\mathbf{B}_{\Lambda_{t}}^{\dagger} \tilde{\mathbf{y}}\right) /\left|\Lambda_{t}\right|,
$$

where $\left|\Lambda_{t}\right|$ is the cardinality of set $\Lambda_{t}$ and counts the number of its elements. Then, the MMSE estimated signal is

$$
\hat{\mathbf{x}}_{\Lambda_{t}}=\left(\mathbf{B}_{\Lambda_{t}}^{H} \mathbf{B}_{\Lambda_{t}}+\frac{\sigma^{2}}{P} \mathbf{I}\right)^{-1} \mathbf{B}_{\Lambda_{t}}^{H} \tilde{\mathbf{y}}
$$

5) Updating the Indices: If there are more than $K$ elements in the identified indices set $\Lambda_{t}$, we propose to update $\Lambda_{t}$ by only retaining the $K$ largest entries in the magnitude of $\hat{\mathbf{x}}_{\Lambda_{t}}$.

Finally, the iterations will stop if the number of iterations is larger than $K$ or if $\left\|\mathbf{r}_{t}\right\|_{2} \geq\left\|\mathbf{r}_{t-1}\right\|_{2}$.

\section{B. Psudocode of the Proposed RSP Algorithm}

Table I shows the procedures of the RSP algorithm.

\section{Performance Evaluation}

\section{A. Simulation Parameters and Performance Metrics}

In the simulation, we assume that $\mathrm{x}$ is a 10 -sparse $256 \times 1$ signal. The amplitudes of the nonzero elements in $\mathrm{x}$ are independent and identically (i.i.d.) uniformly distributed random variables in interval $[c, d]$, with $c=0.4$ and $d=1$. The power of $\mathbf{x}$ is normalized, thus the signal to noise ratio (SNR) in system model (2) is approximated as SNR $\approx 10 \lg ^{1 /\left(1+\frac{N}{M} \sigma^{2}\right)}$. The elements $a_{i j}$ of the $M \times N$ measurement matrix $\mathbf{A}$ are i.i.d. Gaussian variables with $a_{i j} \sim \mathcal{N}(0,1 / M)$. Therefore, each column of $\mathbf{A}$ has unit norm on average.

All the simulations are performed with MATLAB and each simulation result is obtained via $2 \times 10^{4}$ random tests. The codes for OMP, ROMP, SP and CoSaMP are downloaded from the internet. The following two performance metrics are adopted: the first one is the successful recovery rate defined by $\eta=|\hat{\Lambda} \cap \Lambda| /|\Lambda|$ where $\hat{\Lambda}$ is the estimated signal support, the second performance metric is the normalized reconstruction error $\varepsilon=\|\mathbf{x}-\hat{\mathbf{x}}\|_{2} /\|\mathbf{x}\|_{2}$.
TABLE I

The Psudocode of THE Proposed RSP ALgorithm

INPUT: $\mathbf{A}, \mathbf{y}, K, \sigma^{2}$.

DATA PRE-PROCESSING OPERATION:

$\mathbf{Q}=\sigma^{2}\left(\mathbf{I}+\mathbf{A A}^{T}\right) ; \quad \tilde{\mathbf{y}}=\mathbf{Q}^{-1 / 2} \mathbf{y} ; \quad \mathbf{B}=\mathbf{Q}^{-1 / 2} \mathbf{A} ;$

INITIALIZATION:

1) Set the index set $\Lambda_{0}=\emptyset$, the residual $\mathbf{r}_{0}=\tilde{\mathbf{y}}$, the iteration count $t=1$.

2) Calculate the norm of each column of $\mathbf{B}$ by $\boldsymbol{\theta}=\sqrt{\operatorname{diag}\left(\mathbf{B}^{H} \mathbf{B}\right)}$.

3) Initialize the norm of the residual: $\mathbf{n}_{r}(0)=\infty$ and $\mathbf{n}_{r}(1)=\sqrt{\mathbf{r}_{0}^{H} \mathbf{r}_{0}}$.

\section{ITERATIONS:}

WHILE $(t \leq K) \&\left(\mathbf{n}_{r}(t)<\mathbf{n}_{r}(t-1)\right)$

$\%$ Calculate the scaled relevance vector.

$\mathbf{u}_{t}(j)=\boldsymbol{\beta}(j) / \boldsymbol{\theta}(j)$, where $\boldsymbol{\beta}=\mathbf{B}^{H} \mathbf{r}_{t-1} ;$

$\%$ Identify the indices by regularization.

$\left[\mathbf{u}_{t, s}, J\right]=\operatorname{sort}\left(\left|\mathbf{u}_{t}\right|\right.$, 'descend');

$k=1$;

WHILE $(k \leq K) \&\left(\left|\mathbf{u}_{t, s}(k)\right| \geq\left|\mathbf{u}_{t, s}(1)\right| / 2\right)$;

$$
k=k+1 \text {; }
$$

\section{END WHILE}

$J_{0}=J(1: k-1) ; \%$ Obtaining the first $k-1$ elements of $J$.

$\Lambda_{t}=\Lambda_{t-1} \cup J_{0}$

$\%$ Estimate the signal with MMSE method.

$P=\left(\mathbf{B}_{\Lambda_{t}}^{\dagger} \tilde{\mathbf{y}}\right)^{H}\left(\mathbf{B}_{\Lambda_{t}}^{\dagger} \tilde{\mathbf{y}}\right) /\left|\Lambda_{t}\right| ;$

$\hat{\mathbf{x}}_{\Lambda_{t}}=\left(\mathbf{B}_{\Lambda_{t}}^{H} \mathbf{B}_{\Lambda_{t}}+\frac{\sigma^{2}}{P} \mathbf{I}\right)^{-1} \mathbf{B}_{\Lambda_{t}}^{H} \tilde{\mathbf{y}} ;$

$\mathbf{r}_{t}=\tilde{\mathbf{y}}-\mathbf{B}_{\Lambda_{t}} \hat{\mathbf{x}}_{\Lambda_{t}}$;

$\%$ Update the indices by retaining only the $K$ largest elements.

IF $\left|\Lambda_{t}\right|>K$

$\hat{\mathbf{x}}_{t}=\left\{\begin{array}{ll}\hat{\mathbf{x}}_{\Lambda_{t}}, & \text { on the support set } \Lambda_{t} \\ \mathbf{0}, & \text { elsewhere }\end{array} ;\right.$

$\left[\hat{\mathbf{x}}_{s}, J_{s}\right]=\operatorname{sort}\left(\left|\hat{\mathbf{x}}_{t}\right|\right.$, 'descend');

$\Lambda_{t}=J_{s}(1: K)$;

$\%$ Estimate the signal with the updated indices.

$$
P=\left(\mathbf{B}_{\Lambda_{t}}^{\dagger} \tilde{\mathbf{y}}\right)^{H}\left(\mathbf{B}_{\Lambda_{t}}^{\dagger} \tilde{\mathbf{y}}\right) /\left|\Lambda_{t}\right|
$$

$\hat{\mathbf{x}}_{\Lambda_{t}}=\left(\mathbf{B}_{\Lambda_{t}}^{H} \mathbf{B}_{\Lambda_{t}}+\frac{\sigma^{2}}{P} \mathbf{I}\right)^{-1} \mathbf{B}_{\Lambda_{t}}^{H} \tilde{\mathbf{y}} ;$

$\mathbf{r}_{t}=\tilde{\mathbf{y}}-\mathbf{B}_{\Lambda_{t}} \hat{\mathbf{x}}_{\Lambda_{t}}$

ELSE

Continue;

END IF

$\mathbf{n}_{r}(t+1)=\sqrt{\mathbf{r}_{t}^{H} \mathbf{r}_{t}} ;$

$t=t+1$;

END WHILE

\section{OUTPUT:}

The estimated signal $\hat{\mathbf{x}}=\hat{\mathbf{x}}_{t}= \begin{cases}\hat{\mathbf{x}}_{\Lambda_{t}}, & \text { on the support set } \Lambda_{t} \\ \mathbf{0}, & \text { elsewhere }\end{cases}$

and the reconstructed signal support $\hat{\Lambda}=\Lambda_{t}$.

\section{B. Simulation Results}

Compared with the exiting OMP, ROMP, CoSaMP and SP algorithms, the recovery performance of the proposed RSP algorithm is evaluated against the number of measurements $M$ and SNR defined in Section III-A, respectively.

1) Illustration of the noise-folding effect: In this section, the noise-folding effect is demonstrated by comparing the recovery performance of the following two scenarios, i.e., 


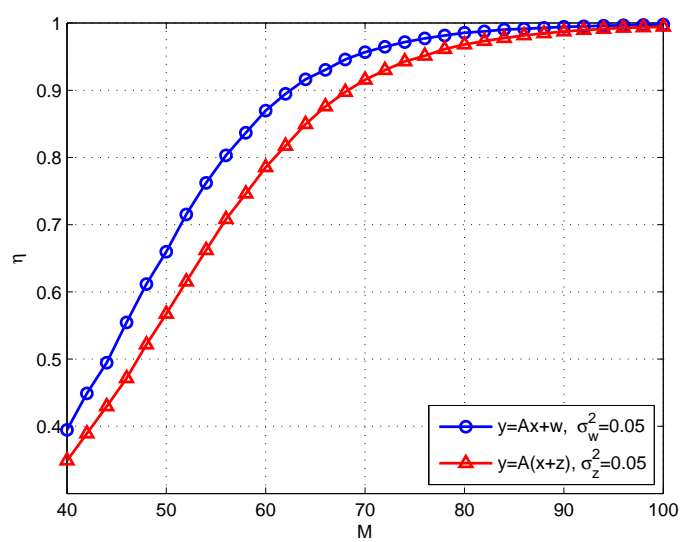

Fig. 1. Illustration of the noise-folding effect, the successful recovery rate $\eta$ vs. the number of measurements $M$.

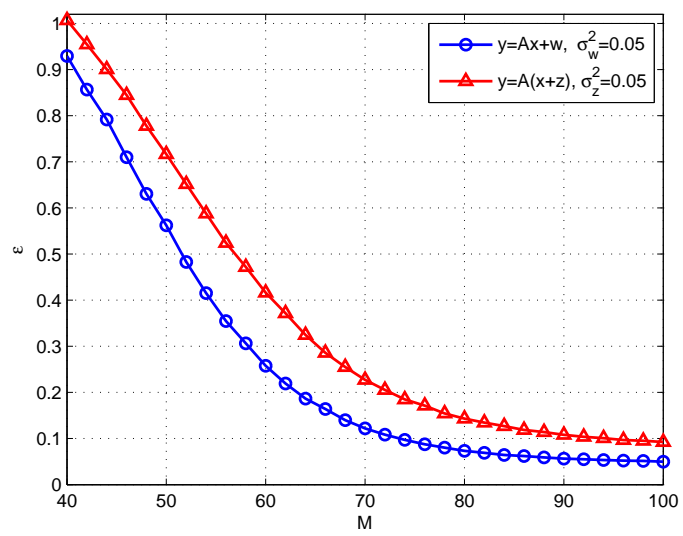

Fig. 2. Illustration of the noise-folding effect, the normalized reconstruction error $\varepsilon$ vs. the number of measurements $M$.

$\mathbf{y}=\mathbf{A}(\mathbf{x}+\mathbf{z})$ and $\mathbf{y}=\mathbf{A x}+\mathbf{w}$, where $\sigma_{z}^{2}=\sigma_{w}^{2}=0.05$. Thus, we have SNR $=13 \mathrm{~dB}$ for $\mathbf{y}=\mathbf{A x}+\mathbf{w}$, and $\mathrm{SNR} \approx$ $10 \lg \frac{M}{12.8} \mathrm{~dB}$ for $\mathbf{y}=\mathbf{A}(\mathbf{x}+\mathbf{z})$. Fig.1 and Fig.2 show that, due to the noise-folding effect, the recovery performance of $\mathbf{y}=\mathbf{A}(\mathbf{x}+\mathbf{z})$ is deteriorated compared with that of $\mathbf{y}=\mathbf{A} \mathbf{x}+\mathbf{w}$. Specifically, Fig.1 indicates that the successful recovery rate of $\mathbf{y}=\mathbf{A}(\mathbf{x}+\mathbf{z})$ is decreased by up to $17 \%$ in comparison with that of $\mathbf{y}=\mathbf{A x}+\mathbf{w}$. Besides, Fig.2 illustrates that the reconstruction error of $\mathbf{y}=\mathbf{A}(\mathbf{x}+\mathbf{z})$ is increased by up to $48 \%$ compared with that of $\mathbf{y}=\mathbf{A x}+\mathbf{w}$.

2) The recovery performance vs. $M$ : Fig. 3 illustrates that, in comparison with OMP, ROMP, CoSaMP and SP algorithms, the proposed RSP algorithm has the highest successful recovery rate $\eta$ with the number of measurements $M$ increasing from 40 to 100 , when $\sigma^{2}=0.05$ and SNR $\approx$ $10 \lg ^{1 /\left(1+\frac{12.8}{M}\right)} \mathrm{dB}$. Specifically, RSP increases $\eta$ by up to $86 \%$, $27 \%$, 48\% and 40\% compared with OMP, ROMP, CoSaMP and SP algorithm, respectively. Fig. 4 shows that, compared with the existing four algorithms, the proposed RSP algorithm has the smallest normalized reconstruction error $\varepsilon$ with the

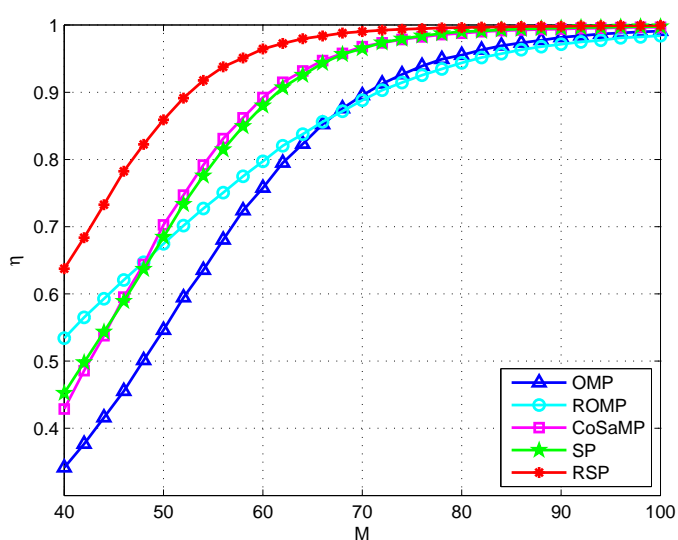

Fig. 3. Successful recovery rate $\eta$ vs. the number of measurements $M$, when $\sigma^{2}=0.05$ and $\mathrm{SNR} \approx 10 \lg ^{1 /\left(1+\frac{12.8}{M}\right)} \mathrm{dB}$.

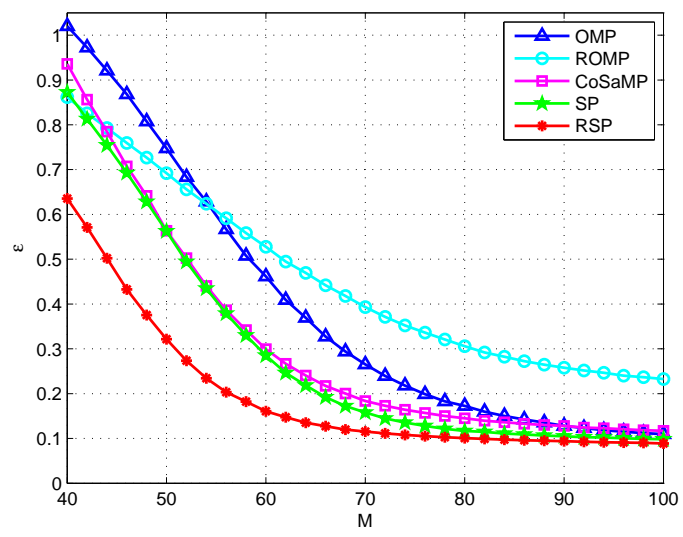

Fig. 4. Normalized reconstruction error $\varepsilon$ vs. the number of measurements $M$, when $\sigma^{2}=0.05$ and SNR $\approx 10 \lg ^{1 /\left(1+\frac{12.8}{M}\right)} \mathrm{dB}$.

increase of $M$. Specifically, the RSP algorithm decreases $\varepsilon$ by up to $65 \%, 71 \%, 47 \%$, and $46 \%$ compared with OMP, ROMP, CoSaMP and SP, respectively.

Besides, Fig. 3 and Fig. 4 demonstrate that, compared with the OMP algorithm, ROMP can improve the recovery performance when $M$ is relative small, but it deteriorates the recovery performance when $M$ is larger, which is not desired. The reason for this phenomenon is that ROMP identifies $2 K$ nonzero elements including at most $K$ correct indices and at least $K$ wrong indices, where the wrong indices deteriorate the recovery performance especially when $M$ is large.

3) The recovery performance vs. SNR: Fig. 5 indicates that, the proposed RSP algorithm has the highest recovery rate $\eta$ in comparison with the existing four algorithms. Specifically, with the increase of SNR, the proposed RSP algorithm increases $\eta$ by up to 50\%, 4\%, 36\% and 34\% compared with OMP, ROMP, CoSaMP and SP algorithms, respectively. Fig. 6 shows that the proposed RSP algorithm has the smallest reconstruction error $\varepsilon$. Specifically, RSP decreases $\varepsilon$ by up to $35 \%, 70 \%, 30 \%$ and $24 \%$ with the increase of SNR compared 


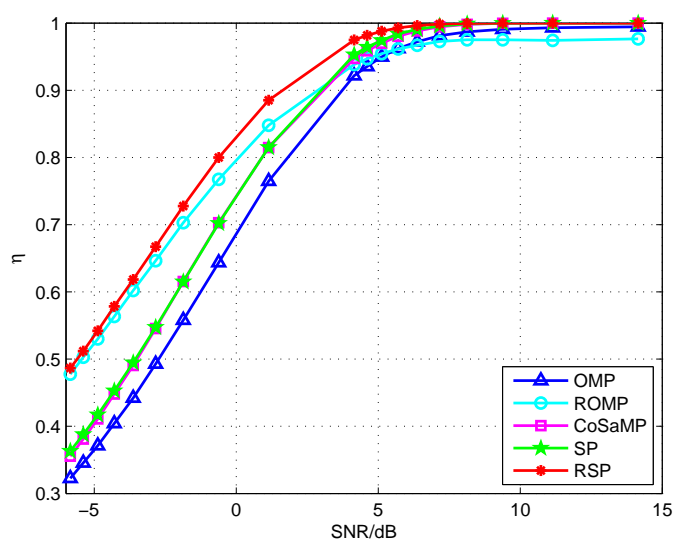

Fig. 5. Successful recovery rate $\eta$ vs. SNR, when $M=90$.

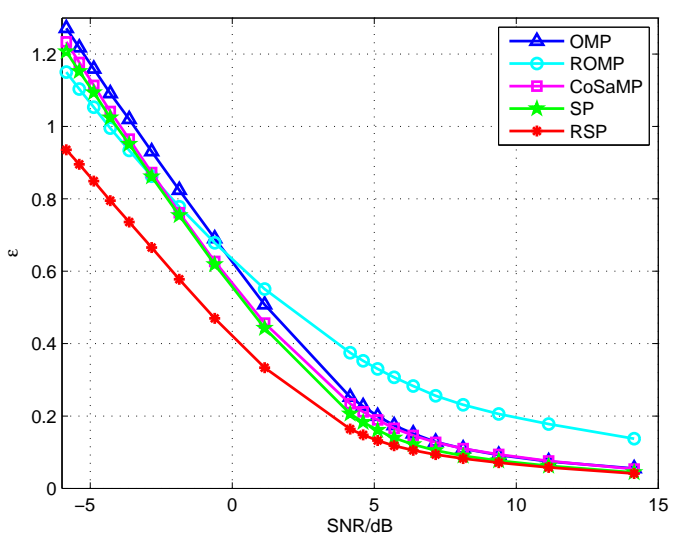

Fig. 6. Normalized reconstruction error $\varepsilon$ vs. SNR, when $M=90$.

with OMP, ROMP, CoSaMP and SP, respectively.

In summary, compared with the ROMP algorithm, the proposed RSP algorithm can simultaneously improve the successful recovery rate $\eta$ and decrease the reconstruction error $\varepsilon$ for all values of the number of measurements $M$ and SNR. Furthermore, compared with the existing OMP, CoSaMP and SP algorithms, the proposed RSP algorithm has the highest $\eta$ and the lowest $\varepsilon$ with the change of $M$ and SNR. The reason that RSP has the best recovery performance is that it simultaneously improves the way of identifying the indices of the nonzero elements and the method of estimating the signal with no more than $K$ updated nonzero elements. Besides, the proposed RSP algorithm alleviates the noise-folding effect via a data pre-processing operation.

\section{CONCLUSION}

To enhance the performance of CS recovery algorithms in the scenarios where both the original sparse signal and the CS measurement vector are contaminated by noise, this paper proposes a Regularized Subspace Pursuit (RSP) algorithm by simultaneously improving the way of identifying the signal support and the method of estimating signals with the updated signal support. Meanwhile, by introducing a data preprocessing operation, the proposed RSP algorithm reduces the noise-folding effect. Simulation results show that, the proposed RSP algorithm has the highest successful recovery rate and the lowest reconstruction error in comparison with the existing OMP, ROMP, CoSaMP and SP algorithms. For example, with the increase of noise variance, the proposed RSP algorithm increases the successful recovery rate by up to $50 \%, 4 \%, 36 \%$ and $34 \%$ (decreases the normalized reconstruction error by up to $35 \%, 70 \%, 30 \%$ and $24 \%$ ) compared with OMP, ROMP, CoSaMP and SP algorithms, respectively.

\section{ACKNOWLEDGEMENTS}

This work was supported by the national Nature Science Foundation of China Project (Grant No. 61001119, 61027003). Besides, Xianjun Yang is a recipient of a Macquarie University Research Excellence Scholarship under a Cotutelle $\mathrm{PhD}$ agreement between Macquarie University and Beijing University of Posts and Telecommunications.

\section{REFERENCES}

[1] D. L. Donoho, "Compressed sensing," IEEE Transactions on Information Theory, vol. 52, no. 4, pp. 1289-1306, 2006.

[2] E. J. Candes and T. Tao, "Near-optimal signal recovery from random projections: Universal encoding strategies?" IEEE Transactions on Information Theory, vol. 52, no. 12, pp. 5406-5425, 2006.

[3] E. J. Cands, "Compressive sampling," in Proceedings of the International Congress of Mathematicians, 2006.

[4] E. J. Candes and T. Tao, "Decoding by linear programming," IEEE Transactions on Information Theory, vol. 51, no. 12, pp. 4203-4215, 2005.

[5] J. A. Tropp, J. N. Laska, M. F. Duarte, J. K. Romberg, and R. G. Baraniuk, "Beyond nyquist: Efficient sampling of sparse bandlimited signals," IEEE Transactions on Information Theory, vol. 56, no. 1, pp. 520-544, 2010.

[6] M. Mishali and Y. C. Eldar, "From theory to practice: Sub-nyquist sampling of sparse wideband analog signals," IEEE Journal of Selected Topics in Signal Processing, vol. 4, no. 2, pp. 375-391, 2010.

[7] E. Arias-Castro and Y. C. Eldar, "Noise folding in compressed sensing," IEEE Signal Processing Letters, vol. 18, no. 8, pp. 478-481, 2011.

[8] J. A. Tropp, "Just relax: convex programming methods for identifying sparse signals in noise," IEEE Transactions on Information Theory, vol. 52, no. 3, pp. 1030-1051, 2006.

[9] Z. Xu, X. Chang, F. Xu, and H. Zhang, "L 1/2 regularization: A thresholding representation theory and a fast solver," IEEE Transactions on Neural Networks and Learning Systems, vol. 23, no. 7, pp. 10131027, 2012.

[10] J. A. Tropp and A. C. Gilbert, "Signal recovery from random measurements via orthogonal matching pursuit," IEEE Transactions on Information Theory, vol. 53, no. 12, pp. 4655-4666, 2007.

[11] D. Needell and R. Vershynin, "Signal recovery from incomplete and inaccurate measurements via regularized orthogonal matching pursuit," IEEE Journal of Selected Topics in Signal Processing, vol. 4, no. 2, pp. 310-316, 2010.

[12] D. Needell and J. A. Tropp, "Cosamp: Iterative signal recovery from incomplete and inaccurate samples," Applied and Computational Harmonic Analysis, vol. 26, no. 3, pp. 301-321, 2009.

[13] D. Wei and O. Milenkovic, "Subspace pursuit for compressive sensing signal reconstruction," IEEE Transactions on Information Theory, vol. 55 , no. 5, pp. 2230-2249, 2009.

[14] T. T. Cai and L. Wang, "Orthogonal matching pursuit for sparse signal recovery with noise," IEEE Transactions on Information Theory, vol. 57, no. 7, pp. 4680-4688, 2011.

[15] R. Wu, W. Huang, and D. Chen, "The exact support recovery of sparse signals with noise via orthogonal matching pursuit," IEEE Signal Processing Letters, vol. 20, no. 4, pp. 403-406, 2013. 